\title{
Layer 5 Corticofugal Projections from Diverse Cortical Areas: Variations on a Pattern of Thalamic and Extrathalamic Targets
}

\author{
${ }^{\circ}$ Judy A. Prasad,* Briana J. Carroll,* and ${ }^{\circledR}$ S. Murray Sherman \\ Department of Neurobiology, University of Chicago, Chicago, Illinois 60637
}

The cerebral cortex, with all its computational power, can only influence behavior via corticofugal connections originating from layer 5 (L5) cells (Sherman and Guillery, 2013). To begin to establish the global pattern of these outputs, we examined L5 efferents originating from four cortical areas: somatosensory, visual, motor, and prefrontal (i.e., ventromedial orbitofrontal) cortex. We injected Cre-dependent adeno-associated virus in an Rbp4-Cre transgenic mouse line (both sexes) to label these L5 efferents selectively. Our study reveals that, across this diverse series of cortical regions, L5 commonly projects to multiple thalamic and extrathalamic sites. We also identified several novel corticofugal targets (i.e., the lateral dorsal nucleus, submedial nucleus) previously unidentified as L5 targets. We identified common patterns for these projections: all areas innervated both thalamus and the midbrain, and all areas innervated multiple thalamic targets, including those with core and matrix cell types (Jones, 1998). An examination of the terminal size within each of these targets suggests that terminal populations of L5 efferents are not consistently large but vary with cortical area and target; and in some cases, these include small terminals only. Overall, our data reveal more widespread and diverse L5 efferents than previously appreciated, suggesting a generalizable role for this cortical layer in influencing motor commands and cognitive processes.

Key words: cortex; corticofugal; corticothalamic; higher order; layer 5; thalamus

\section{Significance Statement}

While the neocortex is responsible for coordination of complex behavior, it requires communication with subcortical regions to do so. It is specifically cortical layer 5 (L5) that is thought to underlie these behaviors, although it is unknown whether this holds true across functionally different cortical areas. Using a selective viral tracing method and transgenic mice, we examined the connectivity of four cortical regions (somatosensory, visual, motor and prefrontal cortex) to assess the generalizability of these L5 projections. All areas of cortex projected to overlapping as well as distinct thalamic and brainstem structures. Terminals within these regions varied in size, implicating that L5 has a broad and diverse impact on behavior.

\section{Introduction}

Regarded as the most highly complex and newest brain region to evolve, the neocortex (hereafter referred to simply as "cortex") greatly increases the brain's computational power and enables

\footnotetext{
Received Mar. 5, 2020; revised June 3, 2020; accepted June 4, 2020.

Author contributions: J.A.P., B.J.C., and S.M.S. designed research; J.A.P. and B.J.C. performed research; J.A.P., B.J.C., and S.M.S. analyzed data; J.A.P., B.J.C., and S.M.S. wrote and edited the paper.

*J.A.P. and B.J.C. contributed equally to this work.

This work was supported by National Institutes of Health Grants NS094184 and NEI EY022388. We thank the University of Chicago's Department of Statistics for the contribution to the design of our terminal size analysis; and Vytas Bindokas (University of Chicago's Integrated Light Microscopy Core) for microscopy guidance.

J. A. Prasad's present address: KickStart Venture Services, University of North Carolina at Chapel Hill, Chapel Hill, North Carolina 27514.

The authors declare no competing financial interests.

Correspondence should be addressed to S. Murray Sherman at msherman@bsd.uchicago.edu.

https://doi.org/10.1523/JNEUROSCI.0529-20.2020

Copyright $\odot 2020$ the authors
}

more flexible control of behavior in response to complex, changing environments. The cortex is typically uninvolved in rote or habitual behaviors, for which more primitive brain areas are recruited. The most primitive of these involve just the spinal circuitry, which allows sensory inputs to directly evoke motor output (Sherrington, 1906). More recently evolved structures, including the brainstem, impose on this spinal system to influence motor output via various bulbospinal pathways. Similarly, when the cortex is involved in behavior, it does so through corticofugal pathways that communicate with lower motor systems (i.e., via the corticobulbar or corticospinal pathways). It is only via these corticofugal outputs that the cortex can communicate with the rest of the central nervous system to affect behavior.

These corticofugal outputs emanate from either layer 5 (L5) or layer 6 (L6) (for review, see Sherman and Guillery, 2013). Based on studies examining the projections from sensory cortices, differences in L5 and L6 pathways suggest distinct functional characteristics. In particular, with rare exceptions, axons from L6 
project only to the thalamus or claustrum (Katz, 1987; Thomson, 2010; Sherman and Guillery, 2013); and since these L6 outputs do not innervate subcortical motor centers, they are unlikely to directly influence behavior. In contrast, axons from L5 innervate certain thalamic nuclei, but they also branch to innervate other brainstem sites that include bulbospinal motor centers and sometimes even spinal cord (Deschênes et al., 1994; Bourassa and Deschênes, 1995; Bourassa et al., 1995; Kita and Kita, 2012; Sherman and Guillery, 2013; Sherman, 2016); see also the Allen Mouse Brain Connectivity Atlas (https://connectivity.brain-map. org/) (Oh et al., 2014). Differences in the thalamic terminal fields derived from L5 and L6 suggest further differences in function: L5 corticothalamic input has been characterized as having driver function, including larger terminals evoking large, depressing EPSPs and activating only ionotropic receptors, whereas the L6 output has been characterized as having modulator function, including smaller terminals activating small, facilitating EPSPs and activating both ionotropic and metabotropic receptors (Sherman and Guillery, 2013; Sherman, 2016). It is thus the L5 efferents that appear to be the route by which cortex influences behavior.

We wished to assess the generality of certain morphologic aspects of the L5 corticothalamic and extrathalamic projections by comparing and contrasting subcortical targets of primary somatosensory cortex (S1), primary visual cortex (V1), primary motor cortex (M1), and the ventromedial orbitofrontal subregion of prefrontal cortex (PFC). We had two objectives: (1) to identify common targets of L5, regardless of cortical origin, and (2) to assess the size of L5 terminals in such targets. Our goal was to identify motifs of L5 cortical efference that may serve to integrate sensory, motor, and cognitive processes. By revealing this structural framework, we believe we have provided a series of over 30 anatomic pathways for future functional exploration.

\section{Materials and Methods}

\section{Experimental design}

Stereotaxic surgery and neuroanatomical techniques. All protocols were approved by the University of Chicago Institutional Animal Care and Use Committee. Transgenic mice were bred by crossing female C57BL6J mice with male $\mathrm{Tg}(R b p 4$-Cre) KL100GSat/Mmcd mice (GENSAT RP24 -285K21) and maintained in a vivarium (conditions: 12 $\mathrm{h}$ light/dark cycle with food and water available ad libitum). Littermates' tail biopsies were genotyped by Transnetyx to confirm mice were Rbp4Cre positive $\left(R b p 4-\mathrm{Cre}^{+}\right)$. This was ensured before performing stereotaxic, Cre-inducible DIO-AAV injections under aseptic conditions.

A total of 13 Rbp4-Cre ${ }^{+}$mice (4 male, 9 female; 70-127 d old) were injected with AAV-EF1a-DIO-hChR2(H134r)-EYFP-WPRE-pA. A total of $3 \mathrm{Rbp} 4$-Cre negative littermates were injected with AAV-hSyn-hChR2 (H134R)-EYFP, serving as negative controls (2 male, 1 female; 70-127 d old). Both viruses were obtained from the University of North Carolina at Chapel Hill Vector Core. Initially, mice were deeply anesthetized using $\sim 3 \%$ isoflurane and head-fixed in a Kopf stereotaxic frame using bite and ear bars. All animals were monitored to ensure depth of anesthesia was maintained throughout surgery $(2 \%-2.5 \%$ isoflurane administered with toe pinches to assess anesthesia level). A unilateral injection was made using a $1 \mu \mathrm{l}$ Hamilton syringe (catalog \#14-824-20, Thermo Fisher Scientific) in 1 of 4 cortical regions for each mouse: S1, V1, M1, or PFC. All stereotaxic coordinates were determined using a stereotaxic atlas (Paxinos and Franklin, 2007) (Table 1). After retracting the scalp, a hand drill was used to expose the injection site for all regions, except for the PFC. For these injections, a craniotomy was performed to expose the sagittal sinus. Volumes were injected at a rate of $\sim 35 \mathrm{nl} / \mathrm{min}$ for each region, resulting in injection times of 11-14 min. Following an additional $10 \mathrm{~min}$ to allow viral diffusion into the site, the syringe was slowly withdrawn, and the scalp incision sutured closed. Both an
Table 1. Stereotaxic coordinates for all cortical Cre-inducible DIO-AAV injections $^{\mathrm{a}}$

\begin{tabular}{lclll}
\hline $\begin{array}{l}\text { Cortical } \\
\text { region }\end{array}$ & $\begin{array}{l}\text { AP coordinates } \\
(\mathrm{mm})\end{array}$ & $\begin{array}{l}\text { ML coordinates } \\
(\mathrm{mm})\end{array}$ & $\begin{array}{l}\text { DV coordinates } \\
(\mathrm{mm})\end{array}$ & $\begin{array}{l}\text { Volume injected } \\
(\mathrm{nl})\end{array}$ \\
\hline S1 $(n=4)$ & -0.90 & \pm 3.1 & -0.5 & 500 \\
V1 $(n=3)$ & 0.50 & \pm 2.5 & -0.5 & 400 \\
M1 $(n=3)$ & 1.54 & \pm 2.0 & -0.5 & 500 \\
PFC $(n=3)$ & 2.46 & N/A & -0.8 & 500
\end{tabular}

${ }^{\mathrm{a}} \mathrm{AP}$ and $\mathrm{ML}$ measurements are from bregma, except for ML coordinates for PFC injections (which were directly on either side of the sagittal sinus), and AP and ML coordinates for V1 injections (which were measured from lambda). All DV coordinates were measured from dura. N/A: not applicable.

antibiotic (Neosporin) and anesthetic (lidocaine hydrochloride) were topically applied to the sutures. The analgesic meloxicam was delivered subcutaneously (1-2 mg/kg dose) postoperatively and once every $24 \mathrm{~h}$ over $2 \mathrm{~d}$.

At 2-3 weeks following the cortical injections, mice were deeply anesthetized with an intraperitoneal injection of ketamine $(100 \mathrm{mg} / \mathrm{kg})$ and xylazine $(3 \mathrm{mg} / \mathrm{kg})$. Each animal was transcardially perfused with 0.025 M PBS followed by fresh 4\% PFA. Brains were extracted and postfixed in $4 \%$ PFA for $24-48 \mathrm{~h}$, then cryoprotected in a $30 \%$ sucrose solution. Once it had sunk, each brain was flash-frozen on dry ice and sectioned coronally using either a cryostat or sliding microtome. Tissue was collected into six series at a thickness of $50-60 \mu \mathrm{m}$ and stored at $-20^{\circ} \mathrm{C}$ in a cryoprotectant solution until they were ready for cytoarchitectonic processing. For each mouse, free-floating sections from a single series were rinsed three times in $0.025 \mathrm{M}$ PBS $(10 \mathrm{~min}$ per wash) and incubated in a $0.1 \mu \mathrm{g} / \mu \mathrm{l}$ solution of DAPI (catalog \#D1306, Thermo Fisher Scientific). Following three 10 min PBS washes, the DAPI-stained series was mounted onto gelatin-coated slides and coverslipped with Vectashield antifade mounting medium (Vector Laboratories). Slides were stored at $4^{\circ} \mathrm{C}$ to preserve the tissue's fluorescent signal.

Microscopy and anatomic screening. All tissue series were examined using a Leica Microsystems upright microscope fitted with fluorescence optics (100 W mercury lamp). Primarily, three filter cubes were used during screening: A4 (excitation $360 \mathrm{~nm}$, emission $470 \mathrm{~nm}$, dichroic $400 \mathrm{~nm}$ ), L5 (excitation $480 \mathrm{~nm}$, emission $527 \mathrm{~nm}$, dichroic $505 \mathrm{~nm}$ ), and TX2 (excitation $560 \mathrm{~nm}$, emission $645 \mathrm{~nm}$, dichroic $595 \mathrm{~nm}$ ). These filters were used to visualize signals for DAPI (blue channel), EYFP (green channel), and autofluorescence (red channel), respectively. Low-power (2.5-40×) photomicrographs were captured using a Retiga 2000 monochrome CCD camera and QCapture imaging software (Teledyne QImaging).

Each series was independently screened by 2 investigators, with labeled terminals identified in each region of interest (ROI) using the cytoarchitectonic DAPI stain and Allen Mouse Brain Reference Atlas (http://mouse.brain-map.org/static/atlas). An ROI was considered a "target" if terminals were observed more than $50 \%$ of subjects within the sample (i.e., 3 of 4 animals for S1 injections and 2 of 3 animals for V1, $\mathrm{M} 1$, and PFC injections). Once targets were identified by each investigator, they cross-referenced findings to ensure there was consistency in target identification and identify potential discrepancies. For simplicity, the ethmoid and posterior medial thalamic nuclei were classified within a single ROI. These targets are presented in Figure 8. To examine terminal morphology, high-power $(63 \times, 1.4 \mathrm{NA})$ photomicrographs of terminals in a subset of thalamic and extrathalamic ROIs were captured using an SP5 2-photon laser scanning confocal microscope (Leica Microsystems). We note that low-power photomicrographs described in each figure are not topographically aligned with adjacent high-power images. Highpower images were chosen as representative of the size of labeled terminals for the nucleus in question (see Quantification and statistical analysis). Low-power images were chosen based on the extent of a terminal field spanning multiple nuclei, which demonstrated the breadth of expression for each injected group.

\section{Quantification and statistical analysis}

Quantification of terminal morphology. Terminal size was quantified using Fiji software (Schindelin et al., 2012) according to the following 

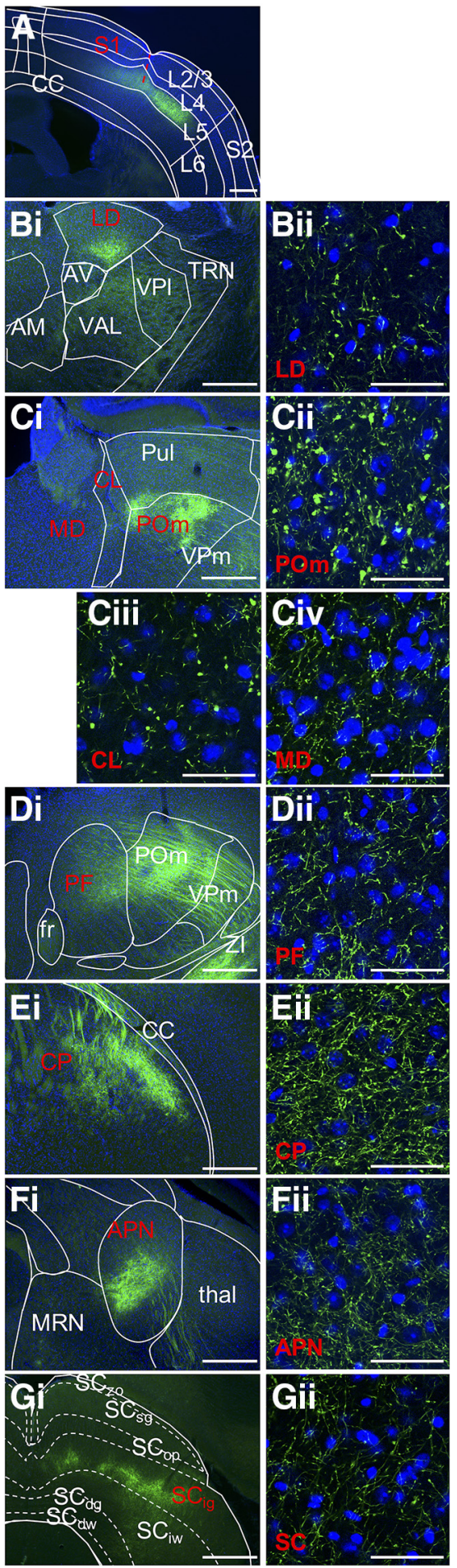

Figure 1. Corticofugal $\mathrm{L} 5$ targets of $\mathrm{S} 1$. Photomicrographs represent coronal sections. Blue represents nuclear stain DAPI. Green represents EYFP label. $\boldsymbol{A}$, S1 injection site where EYFP label is confined to $L 5$, selected thalamic targets are the $(\boldsymbol{B})$ lateral dorsal, $(\boldsymbol{C})$ posterior medial, central lateral, mediodorsal and (D) parafascicular nuclei, and extrathalamic targets $(\boldsymbol{E})$ caudoputamen, $(\boldsymbol{F})$ anterior pretectal nucleus, and $(\boldsymbol{G})$ superior colliculus. For each targeted area, approximate boundaries corresponding to approximate Allen Mouse Brain Reference Atlas panels (http://mouse.brain-map.org/static/atlas), (A) 62, (Bi) 64, (Ci) 73, (Di) 77, (EI) 45 , (Fi) 83, and (Gi) 89, are superimposed on low-power photomicrographs of the right hemisphere. Scale bars, $500 \mu \mathrm{m}$. Dashed red line indicates approximate trajectory of syringe barrel in injection site. Targets labeled in red represent regions for which representative terminal fields are shown under high power in Bii-Gii. Scale bars, $50 \mu \mathrm{m}$. High-power photomicrographs are not topographically matched to low-power images in each case but chosen as representative sections which demonstrate the size of labeled terminals as described automated protocol: The green channel (EYFP signal) was smoothed and thresholded, and terminals were detected as particles with size $\geqq$ $0.25 \mu \mathrm{m}$ and circularity $\geqq 0.3$. This approach was applied to one photomicrograph per animal bearing expression in a given corticofugal target and applied uniformly across all photomicrographs to avoid bias. Terminal sizes were compared by first performing a univariate ANOVA with each injected animal and pathway (defined as the projection from cortical injection site to ROI) as between-subjects variables. The ANOVA was followed by Tukey HSD post hoc tests, in which the Rbp4-Cre+ L5 terminal sizes for a given pathway were compared with Rbp4-Cre negative L6 terminal sizes and with Rbp4Cre+ L5 posterior medial thalamus terminal sizes. Both analyses were performed using SPSS Statistics for Windows, version 22.0 (IBM). With this approach, the terminal size differences between the posterior medial and ventral posteromedial thalamic nuclei following injections in L5 of S1 replicated previous findings (for review, see Sherman and Guillery, 2013) (see Results). We also compared our automated terminal quantification method with traditional manual terminal identification. Specifically, using each method, terminals were identified in a quadrant of the photomicrograph of terminals within the posterior medial nucleus of an S1-injected animal. These resulting populations did not differ in average size (two-tailed $t$ test, assuming equal variance, $p=0.12$ ).

Mixed model statistical test. Using the lme4 package in $\mathrm{R}$ (Bates et al., 2015), we fit a mixed model to the average terminal size in a subset of corticofugal targets. The model allowed fixed effects of cortical injection site and target and a random effect of animal, and fit was assessed with likelihood ratio and bootstrapping. There was no significant effect of animal, but significant effects of site or target were tested in pairwise comparisons (testing the null hypothesis that terminal populations from two cortical sites, or in two subcortical targets do not differ in average size). For these pairwise comparisons, we used a Bonferroni adjustment.

\section{Results}

To assess the distribution and generalization of L5 subcortical targets, we injected four cortical areas, S1, V1, M1, and PFC, with Cre-dependent ChR2-EYFP using the Rbp4-Cre ${ }^{+}$mouse line. These injections produced terminal fields within thalamic nuclei, extrathalamic brainstem sites, and elements of the basal ganglia.

\section{General routes of $\mathrm{L} 5$ corticofugal axons}

We observe consistent patterns of labeled corticofugal axons leaving each cortical area (for representative photomicrographs of injection sites, see Figs. $1 A, 2 A, 3 A, 4 A$ ). Generally, axons travel ventrally from L5 through the corpus callosum and then diverge into two pathways: one bound for the dorsal striatum (specifically, the caudoputamen); and another bound for the internal capsule, which then projects to the brainstem (a route previously described for L5 but not L6 S1 efferents, see Veinante et al., 2000). Below, we describe topographical differences in the

$\leftarrow$

in Figure 7. Sparsely labeled areas may not be evident in low-power images as we aimed to avoid overexposure during acquisition. AV, Anteroventral nucleus; $\mathrm{AM}$, anteromedial nucleus; APN, anterior pretectal nucleus; $\mathrm{CC}$, corpus callosum; $\mathrm{CL}$, central lateral nucleus; $\mathrm{CP}$, caudoputamen; fr, fasciculus retroflexus; LD, lateral dorsal nucleus; MD, mediodorsal nucleus; MRN, midbrain reticular nucleus; PF, parafascicular nucleus; POm, posterior medial nucleus; Pul, pulvinar; $\mathrm{S} 2$, secondary somatosensory cortex; $\mathrm{SC}_{\mathrm{dg}}$ deep gray of the superior colliculus; $\mathrm{SC}_{\mathrm{dw}}$ deep white of the superior colliculus; $\mathrm{SC}_{\mathrm{ig}}$ intermediate gray of the superior colliculus; $\mathrm{SC}_{\mathrm{iw}}$, intermediate white of the superior colliculus; $\mathrm{SC}_{o p}$, optic layer of the superior colliculus; $\mathrm{SC}_{\mathrm{sg}}$ superficial gray of the superior colliculus; $\mathrm{SC}_{20}$, zonal layer of the superior colliculus; TRN, thalamic reticular nucleus; thal, thalamus; VAL, ventral anterior-lateral nucleus; VPI, ventral posterolateral nucleus; VPm, ventral posteromedial nucleus; ZI, zona incerta. 

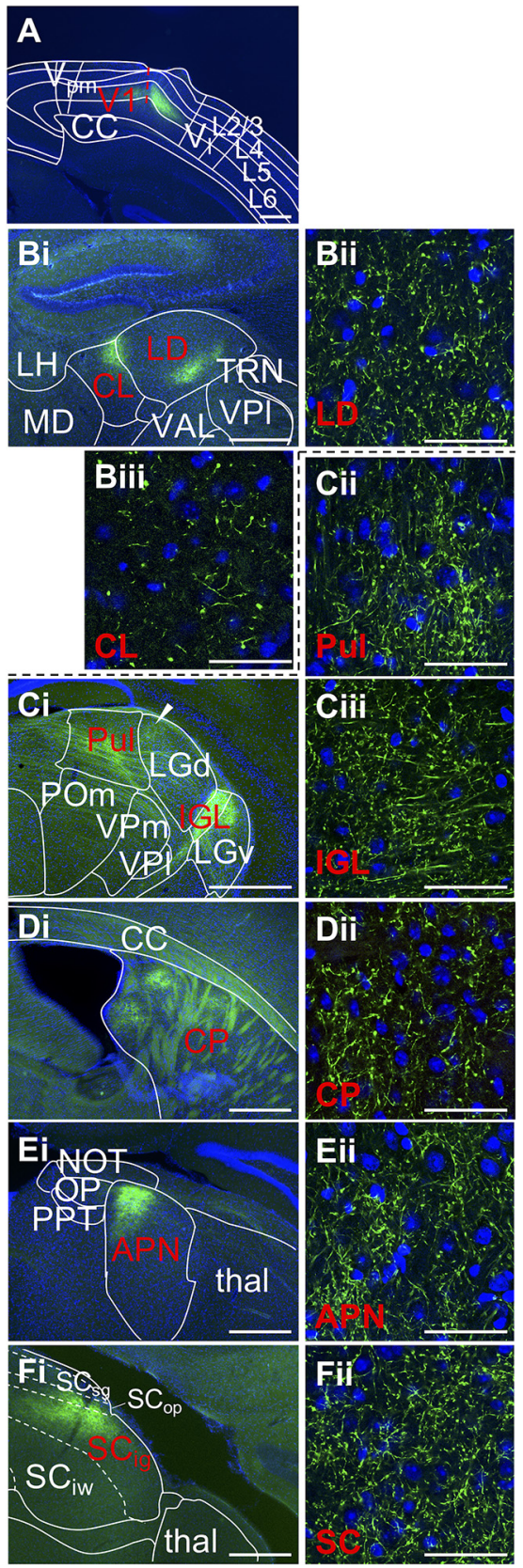

Figure 2. Corticofugal $\mathrm{L} 5$ targets of V1. $\boldsymbol{A}, \mathrm{V} 1$ injection site where EYFP label is confined to $L 5$. Selected thalamic targets are the $(\boldsymbol{B})$ lateral dorsal, central lateral, and pulvinar nuclei and $(\boldsymbol{C})$ intergeniculate leaftlet, and extrathalamic targets $(\boldsymbol{D})$ caudoputamen, $(\boldsymbol{E})$ anterior pretectal nucleus, and $(\boldsymbol{F})$ superior colliculus. Conventions follow Figure 1. Low-power images correspond to Allen Mouse Brain Reference Atlas panels as follows: $(\boldsymbol{A})$ 84, (Bi) 63, and (Ci) 77 , where arrowhead indicates terminals along the border of pulvinar and dorsal lateral geniculate nucleus (Di) 57, (Ei) 81, and (Fi) 87. APN, Anterior prectectal nucleus; CC, corpus callosum; CL, central lateral nucleus; CP, caudoputamen; IGL, intergeniculate leaflet; LD, lateral dorsal nucleus; LGd, dorsal lateral geniculate nucleus; LGv, ventral lateral geniculate nucleus; LH, lateral habenula; MD, mediodorsal nucleus; NOT, nucleus of the optic tract; OP, olivary pretectal nucleus; POm, posterior medial nucleus; PPT, pedunculopontine nucleus; Pul, pulvinar; $\mathrm{SC}_{\mathrm{ig}}$ intermediate gray of the superior colliculus; $\mathrm{SC}_{\mathrm{iw}}$, intermediate white of the superior colliculus; $\mathrm{SC}_{\mathrm{op}}$, optic layer of the superior colliculus; $\mathrm{SC}_{\mathrm{sg}}$, superficial gray of the superior colliculus; thal, thalamus; TRN, thalamic reticular nucleus; VAL, ventral anterior-lateral nucleus; $\mathrm{VI}$, lateral visual cortex; VPI, ventral posterolateral nucleus; VPm, ventral posteromedial nucleus; Vpm, posteromedial visual cortex.
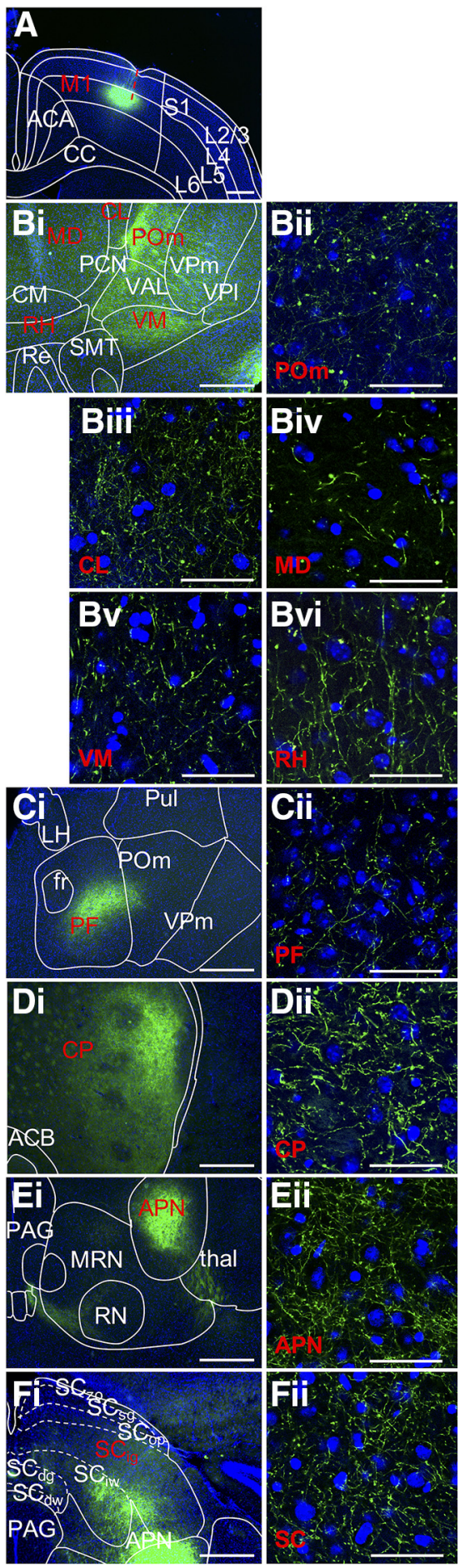

Figure 3. Corticofugal $\mathrm{L} 5$ targets of M1. $\boldsymbol{A}, \mathrm{M} 1$ injection site where EYFP label is confined to $L 5$, selected thalamic targets are the $(\boldsymbol{B})$ posterior medial, central lateral nucleus, mediodorsal, ventral medial, rhomboid and (C) parafascicular nuclei, and extrathalamic targets, $(\boldsymbol{D})$ caudoputamen, $(\boldsymbol{E})$ anterior pretectal nucleus, and $(\boldsymbol{F})$ superior colliculus. Conventions follow Figure 1. Low-power images correspond to Allen Mouse Brain Reference Atlas panels as follows: (A) 46, (Bi) 73, (Ci) 75 (Di) 45, (Ei) 85, and (Fi) 87. ACA, Anterior cingulate cortex; $A C B$, nucleus accumbens; APN, anterior prectectal nucleus; $C$, corpus callosum; $C M$, central medial nucleus; $\mathrm{CL}$, central lateral nucleus; $\mathrm{CP}$, caudoputamen; fr, fasciculus retroflexus; $\mathrm{LH}$, lateral habenula; $M 2$, secondary motor cortex; MD, mediodorsal nucleus; MRN, midbrain reticular nucleus; PAG, periaqueductal gray; $\mathrm{PCN}$, paracentral nucleus; $\mathrm{PF}$, parafascicular nucleus; POm, posterior medial nucleus; Pul, pulvinar; Re, nucleus reuniens; RH, rhomboid nucleus; $\mathrm{RN}$, red nucleus; $\mathrm{SC}_{\mathrm{dg}}$ deep gray of the superior colliculus; $\mathrm{SC}_{\mathrm{dw}}$, deep white of the superior colliculus; $\mathrm{SC}_{\mathrm{ig}}$, intermediate gray of the superior colliculus; $\mathrm{SC}_{\mathrm{iw}}$, intermediate white of the superior colliculus; $\mathrm{SC}_{o p}$, optic layer of the superior colliculus; $\mathrm{SC}_{\mathrm{sg}}$, superficial gray of 

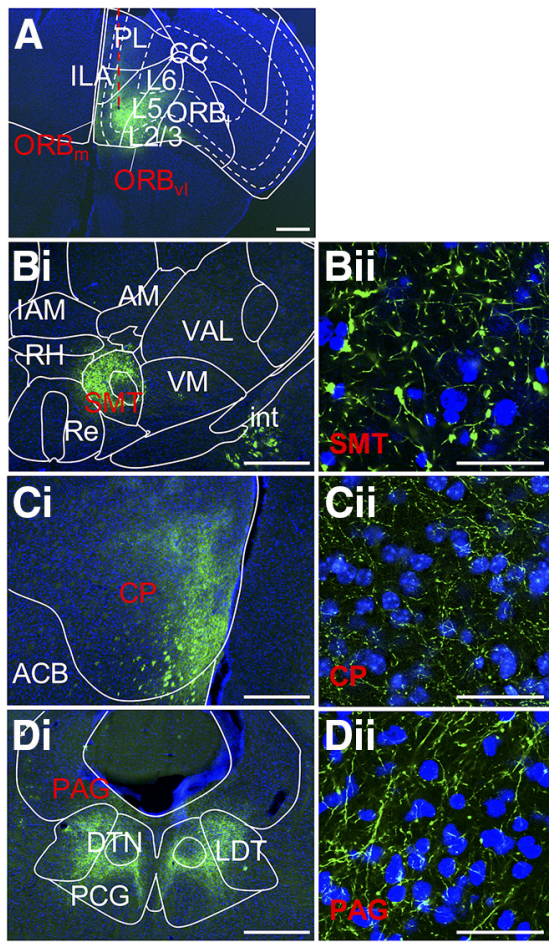

Figure 4. Corticofugal $L 5$ targets of PFC. $\boldsymbol{A}$, PFC injection site where EYFP label is confined to $L 5$. Selected targets are $(\boldsymbol{B})$ thalamic submedial nucleus and extrathalamic targets $(\boldsymbol{C})$ caudoputamen and $(\boldsymbol{D})$ periaqueductal gray. Conventions follow Figure 1. Low-power images correspond to Allen Mouse Brain Reference Atlas panels as follows: (A) 32, (Bi) 65, (Ci) 46, and (Di) 104. ACB, Nucleus accumbens; AM, anteromedial nucleus; $C$, corpus callosum; $C$, caudoputamen; DTN, dorsal tegmental nucleus; IAM, interanteromedial nucleus; ILA, infralimbic cortex; LDT, laterodorsal tegmental nucleus; ORBI, lateral orbital cortex; ORBm, medial orbital cortex; ORBvl, ventrolateral orbital cortex; PAG, periaqueductal gray; PCG, pontine central gray; PL, prelimbic cortex; SMT, submedial nucleus; Re, nucleus reuniens; RH, rhomboid nucleus; VAL, ventral anterior-lateral nucleus; VM, ventral medial nucleus.

distribution of callosal axons in the dorsal striatum (Hintiryan et al., 2016) as well as projections to the internal capsule.

\section{L5 axons from $S 1$}

These axons branch (Fig. 1A), and their collaterals terminate dorsolaterally in the dorsal striatum (Fig. $1 E$ ). We note the terminal fields observed here are interdigitated with unlabeled patches, as previously reported (Tai and Kromer, 2014; Hintiryan et al., 2016). Terminals are also sparsely present in the ventral striatum, specifically, the nucleus accumbens. Axons in the internal capsule pass medial to thalamus primarily around the level of the posterior medial nucleus.

\section{L5 axons from $V 1$}

These axons travel rostrally alongside the optic radiation before diverging subcortically (Fig. 2A). Axons bound for the dorsal striatum continue rostrally, lateral to the ventricle, and synapse along the medial edge of the dorsal striatum (Fig. 2D). Those bound for thalamus pass medially from internal capsule around the level of the intergeniculate nucleus, posterior to the egress of S1 L5 efferents.

$\leftarrow$

the superior colliculus; $\mathrm{SC}_{20}$, zonal layer of the superior colliculus; $\mathrm{SMT}$, submedial nucleus; thal, thalamus; VAL, ventral anterior-lateral nucleus; VM, ventral medial; VPI, ventral posterolateral nucleus; VPm, ventral posteromedial nucleus.
L5 axons from $M 1$

These axons (Fig. 3A) form fields of collaterals on the lateral border of the rostral segment of the dorsal striatum (Fig. 3D). These collaterals are anterior to those observed from injections into S1 and V1, and, similar to the distribution of S1 L5 efferents, are interspersed with unlabeled patches along the lateral border. This terminal field continues past the ventral border of the dorsal striatum, also producing collaterals in the nucleus accumbens. M1 L5 axons bound for thalamus pass medially through the dorsal striatum before joining the internal capsule. These axons exit the internal capsule around the anterior level of the ventral anterior lateral nucleus to reach thalamus.

\section{L5 axons from $P F C$}

Unlike axons from the sensory and motor cortices, axons from PFC (specifically the ventromedial orbitofrontal cortex) traverse ventrally from L5 to terminate in the anterior olfactory nuclei and ventral striatum (Fig. 4A). Traveling via the medial dorsal striatum to reach the internal capsule, PFC L5 axons form collaterals that span the structure's dorsoventral extent. This terminal field additionally crosses the ventral border of dorsal striatum to include the nucleus accumbens (Fig. 4C). We note most of these collaterals were observed anterior to the internal capsule. Axons reaching thalamus leave the internal capsule at a level more anterior to other groups, around the anterior extent of the medial dorsal nucleus.

\section{L5 corticothalamic axons}

Regardless of cortical area injected, our data reveal a series of L5 corticothalamic targets that both encompass and surpass what is noted in the literature.

\section{Thalamic targets of L5 of S1}

While S1 L5 corticothalamic projections to the posterior medial, central lateral, ventral posterolateral, and ventral posteromedial nuclei have been previously described (Bourassa et al., 1995), our data reveal a wider distribution of thalamic targets via two main terminal fields. First, near the anterior level of the posterior medial nucleus, axons traveling ventromedially from the internal capsule give rise to sparse terminals as they course through the ventral posterolateral and ventral anterior lateral nuclei. The major target of these axons is a bilateral terminal field that primarily includes the midline thalamic nuclei reuniens and rhomboid, which receive sparse, diffuse inputs. The second terminal field originates from axons traveling from the caudal aspect of the internal capsule, coursing through ventral posterolateral, ventral posteromedial, and ventral anterior lateral nuclei. Along this route, we observe terminals that vary in density and morphology, and particularly sparsely distributed boutons are most notable on the border between the thalamic reticular nucleus and ventral posterolateral nuclei. We also note isolated clusters of large terminals in the ventral posteromedial nucleus (Liao et al., 2010; Sumser et al., 2017). Some axons travel further medially and continue rostrally to terminate in anterior thalamic nuclei, including the anteromedial and ventral anterior lateral nuclei and the ventral segment of the lateral dorsal nucleus (Fig. 1B). More caudally, terminals densely span the posterior medial, central lateral, and all divisions of the mediodorsal nuclei (Fig. 1C). This terminal field continues dorsally into the pulvinar. More caudally still, this terminal field continues into the parafascicular nucleus (Fig. 1D) and innervates the posterior aspect of the posterior medial nucleus. This labeling presents as a convex arc that spills sparsely into the dorsal region of the subparafascicular 
nucleus and more medially into midbrain structures, including the midbrain reticular nucleus.

Terminal fields span multiple nuclei We regularly observe extensive terminal fields unconstrained by cytoarchitectural boundaries in thalamus and extrathalamic areas following injections in S1, a pattern also observed following other cortical injections described below. This supports the premise that thalamic boundaries may more likely be defined by connectivity than by cytoarchitecture (Sherman and Guillery, 2013; Hunnicutt et al., 2014). Figure 5 provides one such example, in which a field of corticothalamic axons from S1 spans three nuclei: parafascicular, posterior medial, and pulvinar. Although terminals are continuously distributed across these nuclei, there is variation in density and morphology within each. Specifically, terminals in the posterior medial nucleus (Fig. 5, rightmost inset) appear larger than in the parafascicular nucleus (Fig. 5, leftmost inset). This indicates terminal size distributions are not consistently uniform and vary within a terminal field.

\section{Thalamic targets of $L 5$ of $V 1$}

Traveling through the internal capsule, V1 efferents project dorsomedially through the lateral geniculate nucleus, giving rise to two major terminal fields. The more anterior field develops along the border between the pulvinar and dorsal lateral geniculate nucleus (Fig. 2Ci, arrowhead). Two branches of this field reach rostrally to distinct targets (Fig. $2 B$ ): from its dorsal border, a branch reaches medially over the lateral dorsal nucleus to terminate in rostral pulvinar and the dorsal portion of posterior central lateral nucleus; whereas from its ventral border, a branch extends that terminates along the ventrolateral border of the lateral dorsal nucleus and fills this nucleus at its anterior limits. We note that, while L5 efferents from both S1 and V1 target the ventral portion of the lateral dorsal nucleus, there was a clear difference in topographic distribution of the terminals: S1 project primarily ventrally within the nucleus, whereas V1 project primarily ventrolaterally. A separate, more posterior terminal field from V1 densely targets the intergeniculate leaflet. This field is continuous with sparser terminals throughout posterior ventral lateral geniculate nucleus to dorsolateral zona incerta (Fig. 2C).

\section{Thalamic targets of $L 5$ of $M 1$}

Corticothalamic L5 axons from M1 produce four largely separate terminal fields, which include targets previously described (Jeong et al., 2016). Among axons traveling medially, terminals are present throughout the anterior portion of the ventral anterior lateral nucleus, but more caudally, they tend to localize dorsolaterally. En route to this dorsolateral portion of the ventral anterior lateral nucleus, some axons also give rise to terminals in ventral posterolateral and ventral posteromedial nuclei such that a sparse terminal field spans these three nuclei. A second terminal field, slightly posterior to the first, is composed of axons which move more ventrally from the internal capsule to target the border between the ventral anterior lateral nucleus and the ventral medial nucleus. Terminals in this field persist through the posterior extent of the ventral medial nucleus (Fig. 3B). Axons traveling along the same pathway continue medially, passing above or below the medial trigeminal tract to reach a third, very sparse, terminal field distributed across the central medial, and rhomboid nuclei. The fourth and most pronounced field of terminals arises from axons passing dorsally through the ventral anterior lateral nucleus. The anterior limits of the field are in the medial portion of the lateral dorsal nucleus, and the projection continues dorsomedially into the ventral anterior lateral nucleus. More caudally, this field moves ventrolaterally, and emerges squarely in the posterior medial nucleus, where terminals are large and dense. The terminal field spans several medially located nuclei. These include the central lateral and paracentral nuclei, along with the medial, lateral, and caudal divisions of the mediodorsal nucleus where terminals appear less dense relative to the posterior medial nucleus (Fig. 3B). Caudally, the medial portion of this field continues more densely in the dorsolateral parafascicular nucleus, continuous with the field in the posterior medial nucleus. At this level, the field through the parafascicular and posterior medial nuclei is the densest of any thalamic area targeted by M1 L5 (Fig. 3C). Although this field grows sparser at this caudal extent of the thalamus, it persists in the posterior extent of the posterior medial nucleus and subparafascicular nuclei and into the midbrain reticular nucleus. This field (encompassing the posterior medial, parafascicular, subparafascicular, and midbrain reticular nuclei) is similar to a parallel field we observe in our S1 findings but tends to lie ventromedially to it. At this posterior level, the field is joined by axons passing dorsally to midbrain structures.

\section{Thalamic targets of $L 5$ of $P F C$}

L5 axons from the PFC cross the internal capsule and then take two primary routes to the thalamus. The first traverses mediodorsally to produce a very sparse anterior terminal field primarily 

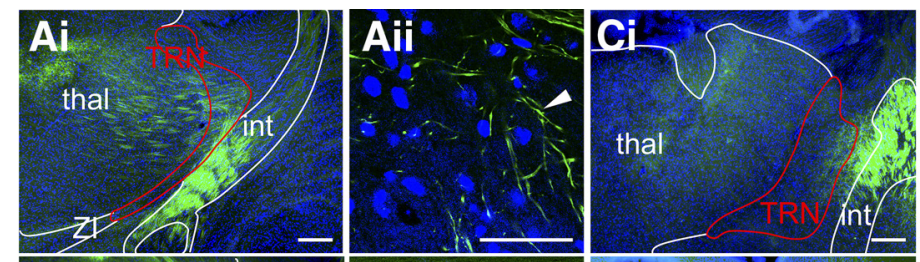

Figure 6. En passant varicosities in the thalamic reticular nucleus: $(\boldsymbol{A})$ from $\mathrm{S1}$, $(\boldsymbol{B})$ from $\mathrm{V} 1,(\boldsymbol{C})$ from $\mathrm{M1}$, and $(\boldsymbol{D})$ from PFC, labeled fibers passing through the thalamic reticular nucleus (red outline) from the internal capsule to the dorsal thalamus. For different injection sites, this passage occurs at different positions along the rostrocaudal axis, corresponding to Allen Mouse Brain Reference Atlas (http://mouse.brain-map.org/static/atlas) (planes 67, 65, 61 and 61, respectively). Scale bars: $\boldsymbol{A}$ Di (low power), $500 \mu \mathrm{m}$; (A-Dii) (high power), $50 \mu \mathrm{m}$. Arrowheads indicate en passant swellings. int, Internal capsule; thal, dorsal thalamus; TRN, thalamic reticular nucleus; Zl, zona incerta.

centered on the parataenial nucleus and then spilling into the paraventricular, central medial and, more caudally, mediodorsal (primarily medial and caudal divisions), paracentral, and central lateral nuclei. Lateral to the parataenial nucleus, across the stria medullaris, terminals are also very sparsely present in the anteroventral nucleus. The second series of axons take a medial route, producing terminals in the lateral part of the ventral anterior lateral nucleus which accompany a much denser terminal field primarily in the submedial nucleus, where orbitofrontal terminals have been previously identified in rat (Alcaraz et al., 2015). Although this field is densest ipsilaterally, the contralateral submedial nucleus is also labeled (more so than other thalamic targets we observed). Terminals in the submedial nucleus are strikingly dense and large, initially filling the nucleus in a single point before creating a ring-like appearance more caudally (where the submedial nucleus encircles the mammillothalamic tract; Fig. 4B). These large terminals spill out of the submedial nucleus into adjacent nuclei, including the anteromedial and ventral medial nuclei and nucleus reuniens. Posterior to these nuclei, terminals sparsely span nucleus reuniens and, to a lesser extent, the rhomboid and subparafascicular nuclei. This terminal field is joined caudally by axons passing through the internal capsule, producing a terminal field in lateral hypothalamus just medial to zona incerta, and continuing dorsomedially through thalamus. Axons move dorsally up the midline toward the ventricle, exhibiting varicosities throughout intralaminar nuclei along this route, including the central medial nucleus, to reach the midbrain.

Cortical L5 inputs target the thalamic reticular nucleus

Among the new, unanticipated corticothalamic targets we identify is the thalamic reticular nucleus. While previous studies have denied the presence of L5 terminals in the thalamic reticular nucleus (Bourassa and Deschênes, 1995; Bourassa et al., 1995), our injections revealed discrete, en passant varicosities of axons crossing the thalamic reticular nucleus from the internal capsule toward other thalamic nuclei (Fig. 6). This medial passage of axons occurs at a different location for each cortical pathway, as described above, creating topographical differences in putative L5 input to the thalamic reticular nucleus. For example, axons from PFC traverse the thalamic reticular nucleus at the most

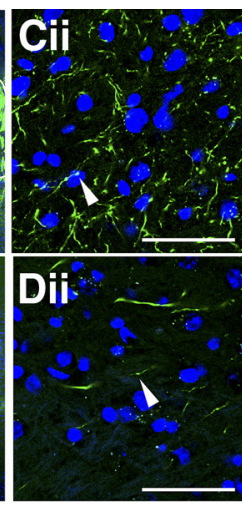

anterior level, with axons from M1 and $\mathrm{S} 1$ crossing through the nucleus caudally. V1 axons traverse across the thalamic reticular nucleus at the most posterior level of any other group.

\section{L5 cortico-extrathalamic subcortical targets \\ Extrathalamic targets of L5 of S1}

Near the posterior aspect of the posterior medial nucleus, S1 L5 axons emerging from the posterior extent of the internal capsule give rise to collateral terminations in the subthalamic nucleus and zona incerta, but also continue to traverse dorsally through posterior thalamus. Axons in the midbrain produce sparse terminals in the midbrain reticular nucleus, ventral tegmental area, and lateral periaqueductal gray, but most densely target the ventral portion of the anterior pretectal nucleus and superior colliculus (Fig. 1F,G). At its anterior aspect, the terminal field in the superior colliculus appears continuous with the adjacent anterior pretectal nucleus and the midbrain reticular nucleus, and is primarily localized within the lateral intermediate gray layer. More caudally, this field continues medially across the superior colliculus and terminates in the intermediate white layer. Remaining axons within the internal capsule extend posteriorly through the cerebral peduncle to the pyramids, providing dense terminals to the pontine gray and tegmental reticular nucleus; they also give rise to axons traveling primarily contralaterally, but also ipsilaterally, to the principal trigeminal nucleus. Terminals are concentrated in the ventral portion of the principal trigeminal nucleus but also present sparsely along its entire perimeter and persist into the spinal trigeminal nucleus. Axons continue to travel posteriorly through the pyramids at this point, which is where we conclude screening.

\section{Extrathalamic targets of $L 5$ of $V 1$}

Projections of V1 L5 to midbrain sites arise from two major radiations. The first emerges from the cerebral peduncle, passing through posterior thalamus to form defined clusters of ramifications in both the dorsal anterior pretectal nucleus (Fig. 2E) as well as superficial layers of the superior colliculus (Fig. 2F). However, we also commonly observe efferents in intermediate layers of the superior colliculus, occasionally reaching deep layers and spilling into dorsal periaqueductal gray. This projection also gives rise to sparse terminals spanning midbrain structures, such as the nucleus of the optic tract, and olivary pretectal nucleus. At the level of the pons arises a second radiation from the internal capsule, which terminates in the pontine gray. These are the most posterior regions considered for this group of animals.

\section{Extrathalamic targets of $L 5$ of $M 1$}

Arising from the caudal portion of the internal capsule, M1 L5 axon bundles pass from the cerebral peduncle to collateralize densely in the ventral portion of the subthalamic nucleus and the zona incerta. Dorsally, these bundles form a loose matrix of terminals that spans the subthalamic and midbrain reticular nuclei. Terminals occasionally and sparsely spill over from the midbrain reticular nucleus into adjacent areas, including the ventral tegmental area and periaqueductal gray. In contrast to the S1 L5 
projections to the anterior pretectal nucleus, M1 L5 targets the anterior pretectal nucleus at a more posterior level. Terminals first appear indistinct from those in the midbrain reticular nucleus on the medial border of the anterior pretectal nucleus, and more caudally, fill the nucleus completely and densely (Fig. 3E). Within the superior colliculus, this projection terminates in intermediate layers but also reaches into deep layers (Fig. $3 F$ ). Nearing the caudal extent of the superior colliculus, the field continues more medially but also remains dense laterally. Another particularly dense group of axons arises from the cerebral peduncle and passes medially under the fasciculus retroflexus, then turns dorsally to form a confined bundle of terminals in the ventral periaqueductal gray and adjacent nuclei. Varicosities are present throughout this route (Fig. $3 E$ ).

At the level of the pons, labeled axons transition from the cerebral peduncle to the pyramids, ramifying in the pontine gray and tegmental reticular nucleus. Axons passing dorsally are bilaterally and sparsely present throughout the pons, including the principal trigeminal nucleus. Patterns of termination here are distinct from those observed in the S1 group: although scattered throughout principal trigeminal nucleus, M1 terminals are densest medial to the nucleus, spilling into the surrounding reticular nuclei while largely avoiding the motor nucleus of the trigeminal nerve. This pattern continues in the spinal trigeminal nucleus, where M1 terminals are present but less densely than in adjacent reticular nuclei.

\section{Extrathalamic targets of PFC L5}

The sparse terminal field produced by PFC axons in the anterior thalamus also continues into the lateral habenula. More caudally, at the posterior extent of the cerebral peduncle, L5 axons from the PFC move into the pyramids, ramifying densely in the pontine gray and tegmental reticular nucleus. Labeled axons arising from the pyramids travel dorsomedially and up the midline, giving rise to terminals en route, including in the midbrain reticular nucleus and ventral tegmental area, reaching the ventral periaqueductal gray, and more caudally, the paracentral gray (a region where label from other injection sites was sparse or absent), where dense terminals surround the embedded dorsal tegmental nucleus (Fig. 4D). We note terminals were also sparsely present in the superior colliculus of one of three injected animals.

\section{Not all corticofugal L5 projections involve large terminals}

Although L5 terminal fields span thalamic boundaries, we also note distinct differences in terminal size between nuclei. Corticothalamic L5 projections have traditionally been associated with larger terminals on average in contrast to smaller terminals from L6 corticothalamic projections (for review, see Sherman and Guillery, 2013; but note diversity within the population including some small L5 terminals as shown by HoerderSuabedissen et al., 2018). To evaluate this difference within our study, we injected Rbp4-Cre negative mice with a non-Cre-dependent virus to induce EYFP expression in S1. These injections labeled terminal projections to the anterior portion of the ventral posteromedial nucleus, which emanate from L6 and not L5 (Killackey and Sherman, 2003; Liao et al., 2010). We then ran a univariate ANOVA, which compared the average terminal sizes from various L5 pathways to Rbp4-Cre negative L6 control data. We found a highly significant overall effect of pathway on terminal size $\left(F_{(25,119868)}=47.83, p<\right.$ $0.001)$. In agreement with prior studies, we found that S1 L5 corticothalamic terminals in the posterior medial nucleus are larger than the S1 terminals in the ventral posteromedial nucleus, primarily from L6 (Tukey HSD post hoc test, $p<0.001$; Fig. 7A).

To compare the L5 terminal sizes beyond the posterior medial and ventral posteromedial nuclei, we measured terminals in a subset of thalamic and extrathalamic targets for each injected cortical group (Fig. 7A). We find that L5 corticothalamic and extrathalamic terminals are larger on average than the primarily L6 terminals in the ventral posteromedial nucleus $(p<0.001$, Tukey HSD post hoc test). Exceptions include S1 L5 terminals in the parafascicular nucleus, M1 L5 terminals in the posterior medial, central lateral, and parafascicular nuclei, and PFC L5 terminals in the central lateral nucleus and dorsal striatum. On average, the largest terminals we observed were those from S1 L5 to POm. However, our analysis identified several additional pathways that were not significantly smaller than this terminal population. Those pathways included S1 L5 terminals in the lateral dorsal nucleus and superior colliculus; V1 L5 terminals in the central lateral and anterior pretectal nuclei; M1 L5 terminals in the medial dorsal, rhomboid, and ventral medial nuclei and dorsal striatum; and PFC L5 terminals in the submedial nucleus and medial dorsal nucleus. Terminal size was most variable in the ventral medial nucleus, but on average fell between that of the posterior medial and ventral posteromedial populations, consistent with Bokor et al., 2016. Collectively, our L5 data suggest that L5 corticothalamic and extrathalamic terminals are not consistently large or small throughout the brain. Rather, these terminals fit within a distribution of sizes that vary depending on cortical origin and target.

While L5 projections are characterized as including both large and small terminal populations, L6 corticothalamic terminals are exclusively small (Van Horn and Sherman, 2004; Llano and Sherman, 2008; Sherman and Guillery, 2013). We confirmed previous observations that the posterior medial nucleus exhibits a larger proportion of large terminals $(>7 \mu \mathrm{m})$ and further observed that the ventral posteromedial nucleus exhibits a preponderance of very small terminals $(<0.5 \mu \mathrm{m})$ (Fig. $7 \mathrm{Bi})$. In thalamus, terminal populations generally resemble the distribution in the posterior medial nucleus. For example, PFC terminals in the submedial nucleus and V1 terminals in pulvinar, where large L5 terminals have been previously described (Bourassa et al., 1995). We also note the varying proportions of large terminals per pathway, such as the projection from V1, which sends fewer large terminals to pulvinar (Fig. 7Bii). Interestingly, S1 and M1 corticothalamic terminals in the parafascicular nucleus (where we observed generally the smallest terminals) lacked large terminals (Fig. 7Biii), suggesting L5 terminals are nonhomogeneous between corticothalamic pathways. Extrathalamic targets from all cortical groups exhibit smaller proportions of both small and large terminals compared with the terminals in the posterior medial nucleus, and, instead, a larger proportion of "intermediate" sized terminals (Fig. 7Biv, arrowhead).

\section{Terminal size correlates with both cortical site and target}

We identified significant effects of cortical site and target on terminal size: pathways originating from M1 had a larger average terminal size than any other group, and PFC smaller, with no significant difference between S1 and V1 (mixed model, $p<0.001$ ). Terminals in the dorsal striatum were significantly larger than in the central lateral, parafascicular, and posterior medial nuclei, the intergeniculate leaflet, the mediodorsal and lateral dorsal nuclei, pulvinar, the ventromedial and rhomboid nuclei, superior colliculus, the anterior pretectal nucleus, and peri- 

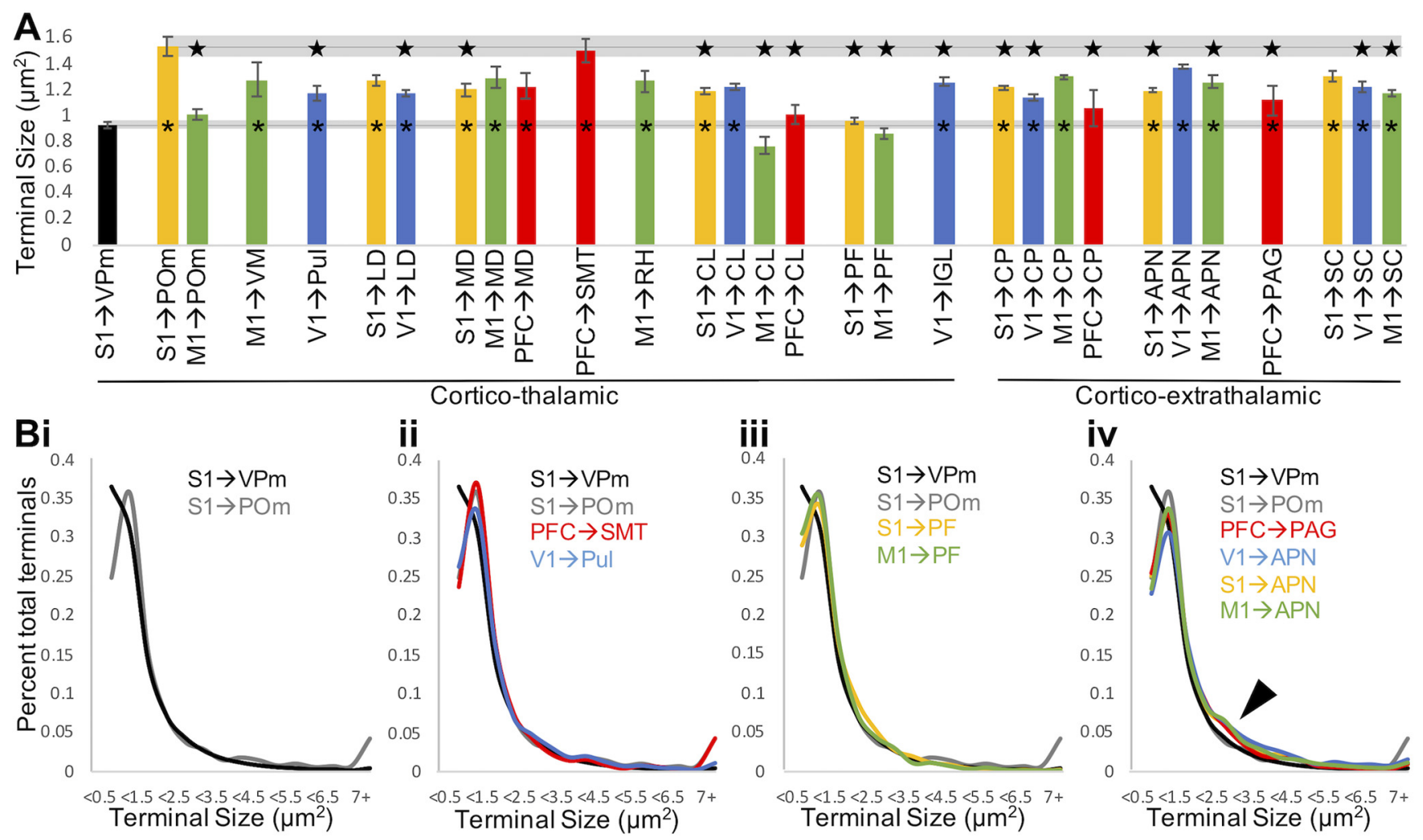

Figure 7. L5 terminal size is pathway-specific. $A$, For a given pathway, bars represent mean terminal size and error bars indicate SEM among animals ( $n=2-4$ per group). For most pathways, average terminal size falls between the small and large terminals established in mouse somatosensory thalamus. The average terminal size in the ventral posteromedial nucleus of Rbp4Cre negative mice and in the posterior medial nucleus of $\mathrm{Cre}^{+}$mice is shown to the left and extended across the graph for easy comparison with these previously established small and large populations. ${ }^{*} p<0.001$, significant difference from terminals in the ventral posteromedial nucleus (ANOVA with animal and pathway as between-subjects variables, Tukey HSD post hoc test). Star represents significant difference $(p<0.001)$ from terminals in the posterior medial nucleus (ANOVA with animal and pathway as between-subjects variables, Tukey HSD post hoc test). $\boldsymbol{B}$, Population distributions of terminal size for pathways of interest. $\mathbf{B i}$, $\mathrm{S} 1$ terminals in the posterior medial nucleus exhibit fewer small and more large terminals than in the posterior medial nucleus. Bii, PFC terminals in submedial nucleus and V1 terminals in the pulvinar exhibit a terminal size distribution similar to S1 terminals in posterior medial nucleus. Biii, S1 and M1 terminals in the parafascicular nucleus exhibit a distribution of small terminals similar to the posterior medial nucleus but, similar to the ventral posteromedial nucleus, lack large terminals. Biv, Terminals in example extrathalamic pathways exhibit more terminals of intermediate size (arrowhead). APN, Anterior prectectal nucleus; $\mathrm{CL}$, central lateral nucleus; $\mathrm{CP}$, caudoputamen; IGL, intergeniculate leaflet; LD, lateral dorsal nucleus; MD, mediodorsal nucleus; PAG, periaqueductal gray; PF, parafascicular nucleus; POm, posterior medial nucleus; Pul, pulvinar; RH, rhomboid nucleus; SC, superior colliculus; SMT, submedial nucleus; VM, ventral medial nucleus; VPm, ventral posteromedial nucleus.

aqueductal gray. Terminals in the superior colliculus were additionally smaller than those in the anterior pretectal nucleus and periaqueductal gray. We conclude that average terminal size varies between pathways, and that this not solely predicted by cortical site or target. Rather, both site and target interact, resulting in terminal size distributions that are pathway-specific.

\section{Discussion}

\section{Summary}

L5 cortical efferents are the sole means by which cortex can effectively influence behavior (Sherman and Guillery, 2013). To identify generalized patterns, we traced L5 outputs from four distinct cortical areas. Our experiments revealed that L5 corticofugal neurons consistently target a wide range of both thalamic and extrathalamic structures, and that the terminals of these axons vary in size related to both the target and cortical area of origin. Common to these patterns is innervation of both thalamus and extrathalamic structures, including the zona incerta, dorsal striatum, pontine gray, and periaqueductal gray (and among all but inconsistently for the PFC group, superior colliculus).

Also common are thalamic targets, which seem to include core- and matrix-dominated nuclei (Jones, 1998). Figure 8 summarizes these projection patterns. By targeting extrathalamic motor areas, L5 corticofugal axons can enact motor commands, and by also targeting thalamus, keep the forebrain informed of these motor commands (Sherman and Guillery, 2013; Sherman, 2016).

Diverse L5 efferents converge in selected subcortical targets Given certain constraints of the Rbp4 mouse line, including a minority of L6 cells which could be labeled with Cre-driven fluorescence (for example photomicrographs, see Harris et al., 2014), we cannot completely exclude the possibility that L6 may contribute to our observed projection patterns. We also acknowledge that not all L5 cells are labeled within the Rbp4-Cre, and that the projections of Cre-negative cells are not captured in our study. In recognition of these limitations, we have only considered subcortical targets identified in the majority of injected animals per group, as these cases provide consistent and reliable outcomes based in the Rbp4-Cre line. Because L5 input defines a thalamic target as higher order (Sherman and Guillery, 2013), the variety of thalamic targets revealed in our study extends our identification of higher-order nuclei. For example, we observed terminals from L5 of S1 in the higher-order posterior medial nucleus, as expected, but also in other thalamic nuclei, including the lateral dorsal, central lateral, and parafascicular nuclei. Likewise, 
terminals from L5 of V1 are not only localized within the pulvinar and lateral dorsal nucleus (for a review of the latter, see Perry and Mitchell, 2019), but also the central lateral nucleus. L5 projections from M1 target several thalamic structures, including the ventromedial, posterior medial, medial dorsal, central lateral, and parafascicular nuclei. The L5 projections from PFC include the thalamic medial dorsal and central lateral nuclei, as well as the submedial nucleus. We further identify extrathalamic L5 terminals from S1, V1, M1, and PFC in the dorsal striatum, and from S1, V1, and M1 in the anterior pretectal nuclei, and superior colliculus. Overall, such considerable overlap in targets of four distinct cortical regions suggests these targets may serve as integrators or hubs of cortical processing.

\section{Novel and unexpected targets receive cortical L5 input}

Of particular interest is our finding of the extensive array of L5 corticothalamic targets, including the thalamic reticular, central lateral, lateral dorsal, and submedial nuclei. Our tracing methodology may explain these new data, as most prior studies exploring L5 projections injected single cortical cells, thereby limiting the extent and variety of labeled corticothalamic cells (Bourassa and Deschênes, 1995; Bourassa et al., 1995; Veinante et al., 2000). Our combination of a L5-specific transgenic mouse line coupled with Cre-dependent viral injections enabled us to identify the extent of L5 corticofugal projections comprehensively relative to the limitations of single-cell labeling. It is thus plausible that novel targets revealed by our injections are innervated by a population of

L5 cells unlabeled in previous studies (Bourassa and Deschênes, 1995; Bourassa et al., 1995; Veinante et al., 2000; Economo et al., 2018). Interestingly, a recent study revealed two separate populations of L5 cells within the anterior lateral motor cortex: one set of axons branches to innervate the thalamus and extrathalamic subcortical sites, whereas the other branches to innervate multiple subcortical sites but not the thalamus (Economo et al., 2018).

\section{The thalamic reticular nucleus receives input from $\mathrm{L5}$ terminals}

A surprising target of L5 input is the thalamic reticular nucleus, in which we observe en passant varicosities from each cortical area injected. We note that this labeling varies topographically with injection site (Fig. 7). Prior studies have explicitly noted that L5 subcortical projections pass through the thalamic reticular nucleus without terminals en route, whereas L6 corticofugal axons do innervate this structure (Bourassa and Deschênes, 1995; Bourassa et al., 1995; Hoerder-Suabedissen et al., 2018; but see Zikopoulos and Barbas, 2006). The robust and consistent fluorescent expression observed throughout each case of our cortical injections supports the validity of our data. Furthermore, the putative terminals observed are nonbranching swellings, presented along the length of the axons which extended through the nucleus. These findings are in stark contrast to the broad ramifications from L6 observed by Bourassa and colleagues (Bourassa 
and Deschênes, 1995; Bourassa et al., 1995). As noted above, these studies used single-cell labeling, which could easily miss unique populations of corticofugal cells (e.g., those that innervate the thalamic reticular nucleus), which may account for the earlier failure to find L5 terminals in the thalamic reticular nucleus. In any case, our light microscopy observations must be further validated through electron microscopy and physiological characterization of these processes to confirm the presence of synaptic terminals.

\section{Implications of the heterogeneity of L5 terminal size}

Our quantification of terminal size demonstrates that, while L5 terminals tend to be large, there is considerable variation in size that is related to both cortical area and target. This carries implications for the physiological impact of L5: in a subset of thalamic nuclei where L5 delivers large terminals, studies of synaptic physiology have indicated a driver phenotype (for review, see Sherman and Guillery, 2013). Only L6 has been reported to deliver to thalamus exclusively small, nondriving terminals that activate metabotropic glutamate receptors. Our data, however, indicate that L5 terminal size is nonhomogeneous within thalamus, and that L5 terminals in some targets are exclusively small (e.g., Fig. 7Biii). This morphologic heterogeneity suggests the possibility of physiological heterogeneity in L5 corticothalamic pathways. Physiologic characterization of these corticothalamic synapses, particularly in target zones that lack large terminals, would be of great interest. This would indicate L5 of cortex has not only a driving influence on subcortical targets, but also potentially a modulatory one. Alternatively, comparisons of terminal sizes between driver and modulator pathways indicate that a main difference is a tail of large terminals associated with the former, and that both pathways have small terminals (Covic and Sherman, 2011; Viaene et al., 2011a,b; Mo and Sherman, 2019); thus, small terminals may still underlie a driver function.

\section{Conclusion}

It is interesting to consider our findings from an evolutionary perspective. Specifically, cortex evolved without coevolution of a motor generation center to which it has unique access. This implies that, to influence behavior via its L5 outputs, cortex must operate through older circuitry in the brainstem and spinal cord. It seems reasonable to assume that, in doing so, cortex would take advantage of circuits more advanced in evolutionary terms rather than having to reinvent the wheel, by directly accessing the oldest circuitry in spinal cord and brainstem. In this regard, the most advanced sensorimotor center in nonmammalian vertebrates is the optic tectum and associated midbrain regions, which remain in mammals major centers for controlling mammalian head and body movements (Gaither and Stein, 1979; Stein and Gaither, 1983; Basso and May, 2017; Suzuki et al., 2019).

From this perspective, we suggest the most efficient way for cortex to influence many or most behaviors is by operating through these brainstem structures. It is particularly interesting that we find sensory, motor, and prefrontal cortical areas exhibit a L5 projection to the midbrain, which is in agreement with other studies (Deschênes et al., 1994; Kita and Kita, 2012; Economo et al., 2018); see also Allen Mouse Brain Connectivity Atlas (https://connectivity.brain-map.org/) (Oh et al., 2014). Furthermore, these studies indicate most or all L5 axons that innervate the midbrain branch to innervate thalamus (Deschênes et al., 1994; Kita and Kita, 2012; Economo et al., 2018); see also Allen Mouse Brain Connectivity Atlas (https://connectivity. brain-map.org/) (Oh et al., 2014). We previously suggested that such branching indicates an exact copy of the message to the midbrain is sent for further cortical processing through the thalamus. To the extent that the message sent to the midbrain by these L5 axons is a motor message, then the copy of that message relayed via thalamus (being an exact copy of a motor message) can, by definition, be read as an efference copy.

The most common motor output of cortex operates via the midbrain, with a copy of that message relayed through thalamus to produce a forward model of the motor command for further cortical processing. However, there is at least one important proviso to this idea. While it seems that all L5 axons that innervate thalamus branch to innervate other subcortical sites as well, not all branching L5 axons innervate thalamus (Bourassa and Deschênes, 1995; Economo et al., 2018). On the other hand, Kita and Kita (2012) labeled 26 L5 corticofugal axons from motor cortex and found that each one had a branch innervating thalamus. Nonetheless, if L5 projections that fail to innervate thalamus do carry motor messages, there is no obvious reciprocal route for an efference copy. One possible explanation for this failure of L5 axons to innervate thalamus is that output from this subset is not a strong driver of motor outputs but plays a more modulatory role not requiring an efference copy. Our work and recent literature highlight the diversity of cortical L5 outputs, but there is clearly much to learn concerning their functional organization and generality across cortex.

\section{References}

Alcaraz F, Marchand AR, Vidal E, Guillou A, Faugère A, Coutureau E, Wolff M (2015) Flexible use of predictive cues beyond the orbitofrontal cortex: role of the submedius thalamic nucleus. J Neurosci 35:13183-13193.

Basso MA, May PJ (2017) Circuits for action and cognition: a view from the superior colliculus. Annu Rev Vis Sci 3:197-226.

Bates D, Machler M, Bolker B, Walker S (2015) Fitting linear mixed-effects models using lme4. J Stat Softw 67:1-48.

Bokor H, Hadinger N, Acsady L (2016) Efficient cortical control of basal ganglia recipient motor thalamus. Program No. 720.18. 2016 Neuroscience Meeting Planner. San Diego, CA: Society for Neuroscience.

Bourassa J, Deschênes M (1995) Corticothalamic projections from the primary visual cortex in rats: a single fiber study using biocytin as an anterograde tracer. Neuroscience 66:253-263.

Bourassa J, Pinault D, Deschênes M (1995) Corticothalamic projections from the cortical barrel field to the somatosensory thalamus in rats: a singlefibre study using biocytin as an anterograde tracer. Eur J Neurosci 7:1930.

Covic EN, Sherman SM (2011) Synaptic properties of connections between the primary and secondary auditory cortices in mice. Cereb Cortex 21:2425-2441.

Deschênes M, Bourassa J, Pinault D (1994) Corticothalamic projections from layer $\mathrm{V}$ cells in rat are collaterals of long-range corticofugal axons. Brain Res 664:215-219.

Economo MN, Viswanathan S, Tasic B, Bas E, Winnubst J, Menon V, Graybuck LT, Nguyen TN, Smith KA, Yao Z, Wang L, Gerfen CR, Chandrashekar J, Zeng H, Looger LL, Svoboda K (2018) Distinct descending motor cortex pathways and their roles in movement. Nature 563:79-84.

Gaither NS, Stein BE (1979) Reptiles and mammals use similar sensory organizations in the midbrain. Science 205:595-597.

Harris JA, Hirokawa KE, Sorensen SA, Gu H, Mills M, Ng LL, Bohn P, Mortrud M, Ouellette B, Kidney J, Smith KA, Dang C, Sunkin S, Bernard A, Oh SW, Madisen L, Zeng H (2014) Anatomical characterization of Cre driver mice for neural circuit mapping and manipulation. Front Neural Circuits 8:76.

Hintiryan H, Foster NN, Bowman I, Bay M, Song MY, Gou L, Yamashita S, Bienkowski MS, Zingg B, Zhu M, Yang XW, Shih JC, Toga AW, Dong HW (2016) The mouse cortico-striatal projectome. Nat Neurosci 19:1100-1114. 
Hoerder-Suabedissen A, Hayashi S, Upton L, Nolan Z, Casas-Torremocha D, Grant E, Viswanathan S, Kanold PO, Clasca F, Kim Y, Molnár Z (2018) Subset of cortical layer $6 \mathrm{~B}$ neurons selectively innervates higher-order thalamic nuclei in mice. Cereb Cortex 28:1882-1897.

Hunnicutt BJ, Long BR, Kusefoglu D, Gertz KJ, Zhong H, Mao T (2014) A comprehensive thalamocortical projection map at the mesoscopic level. Nat Neurosci 17:1276-1285.

Jeong M, Kim Y, Kim J, Ferrante DD, Mitra PP, Osten P, Kim D (2016) Comparative three-dimensional connectome map of motor cortical projections in the mouse brain. Sci Rep 6:20072.

Jones EG (1998) The core and matrix of thalamic organization. Neuroscience $85: 331-345$

Katz LC (1987) Local circuitry of identified projection neurons in cat visual cortex brain slices. J Neurosci 7:1223-1249.

Killackey HP, Sherman SM (2003) Corticothalamic projections from the rat primary somatosensory cortex. J Neurosci 23:7381-7384.

Kita T, Kita H (2012) The subthalamic nucleus is one of multiple innervation sites for long-range corticofugal axons: a single-axon tracing study in the rat. J Neurosci 32:5990-5999.

Liao CC, Chen RF, Lai WS, Lin RC, Yen CT (2010) Distribution of large terminal inputs from the primary and secondary somatosensory cortices to the dorsal thalamus in the rodent. J Comp Neurol 518:25922611.

Llano DA, Sherman SM (2008) Evidence for nonreciprocal organization of the mouse auditory thalamocortical-corticothalamic projection systems. J Comp Neurol 507:1209-1227.

Mo C, Sherman SM (2019) A sensorimotor pathway via higher-order thalamus. J Neurosci 39:692-704.

Oh SW, Harris JA, Ng L, Winslow B, Cain N, Mihalas S, Wang Q, Lau C, Kuan L, Henry AM, Mortrud MT, Ouellette B, Nguyen TN, Sorensen SA, Slaughterbeck CR, Wakeman W, Li Y, Feng D, Ho A, Nicholas E, et al. (2014) A mesoscale connectome of the mouse brain. Nature 508:207214.

Paxinos G, Franklin KB (2007) Mouse brain in stereotaxic coordinates. San Diego: Elsevier Academic.

Perry BAL, Mitchell AS (2019) Considering the evidence for anterior and laterodorsal thalamic nuclei as higher order relays to cortex. Front Mol Neurosci 12:167.
Schindelin J, Arganda-Carreras I, Frise E, Kaynig V, Longair M, Pietzsch T, Preibisch S, Rueden C, Saalfeld S, Schmid B, Tinevez JY, White DJ, Hartenstein V, Eliceiri K, Tomancak P, Cardona A (2012) Fiji: an opensource platform for biological-image analysis. Nat Methods 9:676-682.

Sherman SM (2016) Thalamus plays a central role in ongoing cortical functioning. Nat Neurosci 19:533-541.

Sherman SM, Guillery RW (2013) Functional connections of cortical areas: a new view from the thalamus. Cambridge, MA: Massachusetts Institute of Technology.

Sherrington CS (1906) Observations on the scratch-reflex in the spinal dog. I Physiol 34:1-50.

Stein BE, Gaither NS (1983) Receptive-field properties in reptilian optic tectum: some comparisons with mammals. J Neurophysiol 50:102-124.

Sumser A, Mease RA, Sakmann B, Groh A (2017) Organization and somatotopy of corticothalamic projections from L5B in mouse barrel cortex. Proc Natl Acad Sci USA 114:8853-8858.

Suzuki DG, Pérez-Fernández J, Wibble T, Kardamakis AA, Grillner S (2019) The role of the optic tectum for visually evoked orienting and evasive movements. Proc Natl Acad Sci USA 116:15272-15281.

Tai AX, Kromer LF (2014) Corticofugal projections from medial primary somatosensory cortex avoid EphA7-expressing neurons in striatum and thalamus. Neuroscience 274:409-418.

Thomson AM (2010) Neocortical layer 6, a review. Front Neuroanat 4:13.

Van Horn SC, Sherman SM (2004) Differences in projection patterns between large and small corticothalamic terminals. J Comp Neurol 475:406-415.

Veinante P, Jacquin MF, Deschênes M (2000) Thalamic projections from the whisker sensitive regions of the spinal trigeminal complex in the rat. J Comp Neurol 420:233-243.

Viaene AN, Petrof I, Sherman SM (2011a) Properties of the thalamic projection from the posterior medial nucleus to primary and secondary somatosensory cortices in the mouse. Proc Natl Acad Sci USA 108:1815618161.

Viaene AN, Petrof I, Sherman SM (2011b) Synaptic properties of thalamic input to the subgranular layers of primary somatosensory and auditory cortices in the mouse. J Neurosci 31:12738-12747.

Zikopoulos B, Barbas H (2006) Prefrontal projections to the thalamic reticular nucleus form a unique circuit for attentional mechanisms. J Neurosci 26:7348-7361. 